Service-Center für

Hörimplantat-Systeme

Im Hörzentrum Hannover (nahe der Medizinzischen Hochschule Hannover) hat MED-EL Medical Electronics, einer der führenden Anbieter von Hörimplantaten ein Beratungs- und Servicebüro eröffnet. Seit Anfang Mai können sich dort Patienten sowie alle an Hörimplantatsystemen Interessierte professionell beraten lassen und umfassende Serviceleistungen in Anspruch nehmen. Das neue MED-EL Care Center ${ }^{\mathrm{Tm}}$ bündelt alle nötigen Kompetenzen und bietet die Vorteile kurze Wege und effizienter und reibungsloser Abläufe. Alle für die Beratung, Anpassung und Rehabilitation notwendigen Schritte können zentral im Hörzentrum erledigt werden.

Nach Informationen von MED-EL, Starnberg

\section{Fliegen ohne Druck} im Ohr

Bei rund einem Drittel aller Flugreisenden macht sich der schnell ansteigende Kabinendruck während des Landeanflugs in den Ohren unangenehm oder sogar schmerzhaft bemerkbar. Denn bei der Überwindung großer Höhenunterschiede in kurzer Zeit können die Eustachischen Röhren den Druckausgleich nicht schnell genug herstellen. Vor allem für kleine Kinder, die die ValsalvaTechnik noch nicht beherrschen, kann dies zum Problem werden. Mit zwei Spezialohrstöpseln namens earPlanes ${ }^{\circledR}$ lassen sich diese unschönen Begleiterscheinungen des Fliegens einfach vermeiden. Sie bestehen aus hypoallergenem Silikon mit einem Hohlraumfilter aus Keramik, der die ein- und ausströmende Luft reguliert. So werden starke Druckschwankungen aufgefangen und der schmerzhafte Druck auf das Trommelfell vermieden. earPlanes ${ }^{\circledR}$ sind für Erwachsene und in einer kleineren Version für Kinder in der Apotheke erhältlich. (unverbindliche Preisempfehlung 8,15 Euro pro Paar).

Nach Informationen von Cirrus Healthcare

\section{Impftablette mit Langzeiteffekt}

— Dass die zur Behandlung von Gräserund Roggenpollenallergie eingesetzte spezifische Immuntherapie mit der „Impftablette“ Grazax ${ }^{\circledR}$ auch noch zwei Jahre nach Therapieende Wirkung zeigt, ergab nun die GT-o8-Studie, deren Ergebnisse kürzlich auf dem Europäischen Kongress für Allergologie, Asthma und klinische Immunologie in London erstmals vorgestellt wurden. „Zwei Jahre nach Abschluss der Behandlung konnten wir beobachten, dass Grazax ${ }^{\circledR} \mathrm{Heu}$ schnupfensymptome im selben Ausmaß reduziert wie im Vorjahr der klinischen Studie. Mit dem Abschluss der GT-08-Studie haben wir jetzt mehr Nachweise, dass die Wirksamkeit während und auch noch zwei Jahre nach der Behandlung mit GRAZAX ${ }^{\circledR}$ erhalten bleibt“, betonte Prof. Stephen R. Durham, Leiter der GT-o8-Studie und Leiter des Bereichs Allergologie und Klinische Immunologie am National Heart \& Lung Institute, Imperial College London. Damit erfüllt Grazax ${ }^{\circledR}$ die Forderung der Europäischen Arzneimittel-Agentur (EMEA) nach robusten klinischen Daten zur spezifischen

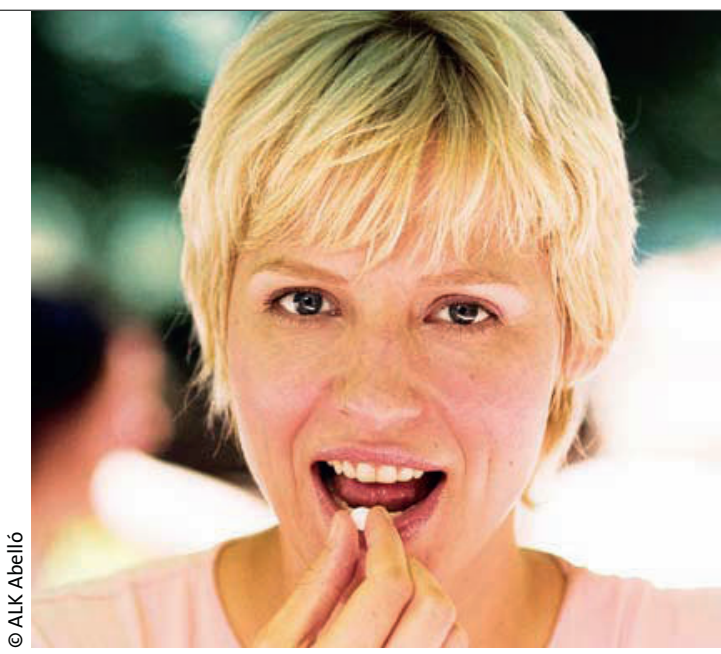

Auch zwei Jahre nach Abschluss der Therapie zeigte die „Impftablette“ Wirkung.

Immuntherapie, nach Langzeitwirksamkeit und nach einem anhaltenden klinischen Effekt während und nach Abschluss der Behandlung. Möglicherweise bringen die Studienergebnisse einen Durchbruch bei der künftigen Therapie der durch Gräserpollen ausgelösten allergischen Rhinokonjunktivitis. Die Immuntherapie $m+i t$ Grazax ${ }^{\circledR}$ kann im Übrigen auch intrasaisonal begonnen werden.

gz

Nach Informationen von Alk Abelló, Wedel

\title{
Pflanzenpräparate bei verstopften Nasennebenhöhlen
}

- Bei Rhinosinusitis sind Antibiotika nur sinnvoll, wenn eitriges Nasensekret auf eine bakterielle Infektion hinweist und diese mit starken Beschwerden oder erhöhtem Komplikationsrisiko verbunden ist. Ansonsten sprechen die meist viralen Infektionen gut auf eine Therapie mit pflanzlichen Kombipräparaten an, wie Dr. Andreas Schapowal, Landquart/Schweiz, kürzlich erläuterte.

Ein bewährtes Kombinationspräparat bei akuter Rhinosinusitis ist das Phytotherapeutikum Sinupret ${ }^{\circledR}$. Es enthält den Kombinationswirkstoff BNO 101 aus Eisenkraut, Enzian, Sauerampfer, Holunder und Schlüsselblume. Er wirkt bei akuten und chronischen Entzündungen der Nasennebenhöhlen sekrolytisch-antiobstruktiv und hat antiinflammatorische, antimikrobielle und immunmodulierende Effekte. Wirksamkeit und Verträglichkeit des Kombipräparates sind in mehreren placebokontrollierten Studien und in einer Metaanalyse mit 900 Patienten dokumentiert. Letztere ergab relevante Behandlungsvorteile für die Phytotherapie, auch erkennbar als Besserung im Röntgenbefund und bei der Belüftung der Nasennebenhöhlen. Das Patienten-Votum fiel klar zugunsten der pflanzlichen Therapie aus. Bei unkomplizierten Erkältungskrankheiten bringen qualitativ hochwertige Phytotherapeutika sehr gute Therapieerfolge, so das Fazit des HNO-Arztes. Dabei seien die pflanzlichen Präparate genauso gut verträglich wie Placebo und allergische Reaktionen selten.

bg

Symposium zur 81. Jahresversammlung der Deutschen Gesellschaft für Hals-Nasen-

Ohren-Heilkunde, Kopf- und Hals-Chirurgie e.V. Wiesbaden, 14. Mai 2010. Veranstalter: Bionorica AG, Neumarkt 